\title{
Effects of plies orientations and initial geometric imperfections on buckling strength of a composite stiffened panel
}

\author{
Ikram Feddal, a , Abdellatif Khamlichi ${ }^{2}$ and Koutaiba Ameziane ${ }^{1}$ \\ ${ }^{1}$ Physics of Condensed Matter Laboratory, Faculty of Sciences, Tetouan 93002, Morocco \\ ${ }^{2}$ Systems of Communication and Detection Laboratory, ENSA Tetouan, 93030 Tetouan, Morocco
}

\begin{abstract}
The use of composite stiffened panels is common in several activities such as aerospace, marine and civil engineering. The biggest advantage of the composite materials is their high specific strength and stiffness ratios, coupled with weight reduction compared to conventional materials. However, any structural system may reach its limit and buckle under extreme circumstances by a progressive local failure of components. Moreover, stiffened panels are usually assembled from elementary parts. This affects the geometric as well as the material properties resulting in a considerable sensitivity to buckling phenomenon. In this work, the buckling behavior of a composite stiffened panel made from carbon Epoxy Prepregs is studied by using the finite element analysis under Abaqus software package. Different plies orientations sets were considered. The initial distributed geometric imperfections were modeled by means of the first Euler buckling mode. The nonlinear Riks method of analysis provided by Abaqus was applied. This method enables to predict more consistently unstable geometrically nonlinear induced collapse of a structure by detecting potential limit points during the loading history. It was found that plies orientations of the composite and the presence of geometric imperfections have huge influence on the strength resistance.
\end{abstract}

\section{Introduction}

In various fields of engineering, composite stiffened panels are used mainly due to their high strength-toweight and stiffness-to-weight ratios. They have also good impact energy and sound absorption, and often low production cost. However, every structure is prone to stability problems and buckling before it reaches the geometrically linear strength limit. The global or the local buckling phenomena may occur and lead to the collapse of the structure well before that ideal limit load. In general, buckling happens when slender structures such plates or beams are subject to specific loading patterns such as an axial load applied in compression. Stiffened panels made by assembling columns and shells constitute an optimized configuration which is intended to improve resistance to buckling. For a secure design, it is important to understand the buckling behaviour and to relate it to geometric and material properties as well as the various imperfections affecting the structure. Geometric imperfections include initial imperfections related to the fabrication process. They can be distributed or localized such as initial distortions resulting from assembling process or impact happening during service life.

From the mechanical point of view, buckling of composite stiffened panels is very intricate and involves complex interactions. Various methods have been proposed to tackle this issue. They are based on experimental, numerical and computational approaches. The critical load of laminated composite plates as calculated by a linear buckling analysis was recognized to under-estimates the load-carrying capability of the system. Laminated composite plates possess loadcarrying capacity after buckling which is generally several times that of the critical buckling load. Thus, the real practical limits of the load-carrying capability can be determined only by a nonlinear step-by-step analysis where the post-buckling behaviour is considered. This is termed also the strength resistance analysis of the structure.

Considerable efforts have been made for the numerical analysis of the buckling and post-buckling analysis over the years. Many studies have been dedicated to investigate the buckling related phenomena in the case of composite structures. Leissa [1] gave a review of the buckling and postbuckling studies of composite laminated plates up to 1986, and then he reviewed the development of buckling analysis of laminated composite plates with linear effective constitutive properties. Zhang and Yang [2] gave a review of the developments achieved for laminated composite plates between 1990 and 2009 and based on the finite element analysis. This literature review included buckling and post-buckling analysis of composite laminated plates. The buckling and post-buckling behaviour of unidirectional and cross-ply composite laminated plates with multiple delaminations has been studied in [3].

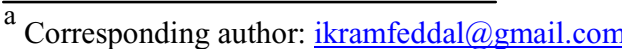


Non-linear finite elements analyses have been performed by using an incremental-iterative method. Large displacements and contact constraints between sublaminates have been taken into account. The authors found that delamination length and position as well as stacking sequence of the plies influence largely the critical load of the plate. More recently, buckling and post-buckling of thick composite plates having large initial geometrical imperfections have been studied in [4]. The authors have used the theory of large transformations based on the second Piola-Kirchhoff stress and Green-Lagrange strain and considered plates exhibiting large initial imperfections. Liang and Sun [5] used a novel reduced-order modelling strategy based on the Koiter-Newton method to analyse buckling and postbuckling of laminated composite plates with delamination. Various laminated plates with different thicknesses, delamination lengths and stacking sequences were considered and enabled to validate the good performance of the method in terms of numerical accuracy and performance. Namdar and Darendeliler [6] investigated buckling, post-buckling and progressive failure of composite laminated plates by using both numerical and experimental methods. Buckling load, load-displacement relations for post buckling and maximum out-of-plane displacements of the plates were determined. The numerical results were compared with experimental findings for different laminates. The buckling resistance was found to depend largely on the orientation of plies with regards to the compressive load. A good agreement between numerical and experimental results was obtained for buckling load and post-buckling behaviour especially for the laminates with unidirectional tapes. Heidari-rarani and Khalkhali-sharifi [7] investigated the buckling behaviour of symmetric balanced square laminated composite plates theoretically and experimentally. The obtained results showed that failure starts from the plate edges and develops along the plate width. Using a numerical study on $[ \pm \theta] \mathrm{s}$ and $[\theta / \theta+90]$ s layups, they showed that by increasing the $\theta$ from 0 to 90 , the critical buckling load decreases continuously. Dawn [8] conducted a study on the buckling of laminated plates where he has considered different ply orientation sets with different thickness for each ply. Using a refined shear theory, he found that the buckling load is sensitive to the ply orientation and that the $-/+45$ ply orientation is the most sensitive to transverse shearing taking place in laminates plates. Langvarsky and Bocko [9] investigated the buckling of composite plates having different orientations of layers. The authors used finite element based analysis in Abaqus CAE. They considered four different orientations with three, six and twelve layers loaded by shear and compression loads. They identified the inappropriate orientations of layers for different type of loads. They noticed that the orientations of composite layers affect the value of the critical buckling load. In particular, the composite plates with three layers and orientation of layers $[90,0,90]$ was found to be more sensitive to compression load than shear load.
Considering buckling of laminated composite plates with delamination, interaction between the delamination and the buckling strength exists. Growth of delamination was related to the total strain-energy release $\mathrm{G}$ by a technique based on a virtual crack closure, Whitcomb and Shivakumar [10]. The method enabled to predict a large variation of $G$ along the delamination front for square and rectangular delaminations. Self-similar growth is then not expected to occur as a delamination may grow in the load direction or perpendicular to the load direction. This depends on the current delamination aspect ratio, the strain level, and the absolute size of the delamination.

In the framework of the following analysis, delamination is assumed to be stationary and not affected by the actual state of loading. The buckling behaviour of a stiffened composite panel is studied by means of finite element modelling under Abaqus software package. The panel is discretized by using special shell elements, which allows defining the material of composite panel, the thickness and layout of layers in the composite stiffened panel. We consider a carbon Epoxy Prepreg stiffened panel with different plies orientations sets. The initial distributed geometric imperfection is taken into account in order to assess its influence on the buckling strength. This has the form of the first Euler buckling mode which is determined by a pre-analysis. The geometrically nonlinear problem is solved by using Riks Algorithm. The limit load is identified as that for which the related load increment changes the sign.

\section{Materials and methods}

\subsection{Geometry and initial imperfection}

Geometric nonlinearities are considered while the material is assumed to have a linear elastic behaviour. The simulation is done by means of the Abaqus software and the famous S4R shell element is used. This element has four nodes with six degrees of freedom at each node. It can consider both material and geometric nonlinearities. In the present analysis, the geometrical configuration of the stiffened panel is fixed and the boundary conditions that were considered in the numerical simulations are represented as shown in figure 1. The displacements are designated by $\mathrm{u}$ and the rotations by $\theta$.

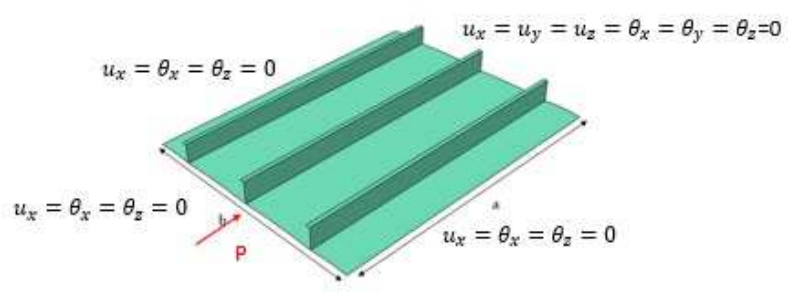

Figure 1. The geometric configuration of the stiffened panel and the considered boundary conditions. 
The total length of the plate is a $=958 \mathrm{~mm}$ and the width is $b=757.5 \mathrm{~mm}$. The stiffeners take the shape of $\mathrm{L}$ with a fixed thickness $t_{w}=2.95 \mathrm{~mm}$ supposed uniform, their height is $h_{w}=64 \mathrm{~mm}$, the thickness of the flange is $t_{\mathrm{f}}=4.3 \mathrm{~mm}$ and its height is $b_{\mathrm{f}}=12 \mathrm{~mm}$, see figure 2 .

The skin plate of the stiffened panel is supposed to be distorted by initial imperfections due to the assembling process. This distortion is assumed to be proportional to the first Euler buckling mode of the stiffened panel. This is provided by performing a linear buckling analysis. The following eigenvalue problem is solved to get the buckling load multipliers $\lambda_{\mathrm{i}}$ and buckling modes $\psi_{\mathrm{i}}$

$$
\left([\mathrm{K}]+\lambda_{\mathrm{i}}[\mathrm{S}]\right)\left\{\psi_{\mathrm{i}}\right\}=0
$$

where $[\mathrm{K}]$ is the stiffness matrix and $[\mathrm{S}]$ is the stress stiffness matrix. The stress stiffness matrix is computed based on the initial stress state induced by the applied load.

The first buckling mode is obtained by using an algorithm available under Abaqus that extracts the buckling modes $\psi_{\mathrm{i}}$ associated to the previous eigenvalue problem.

The initial geometric imperfection is then defined by taking the maximum amplitude of distortion equal to $\mathrm{w}_{0}$. The first bucking mode is given in figure 2 .

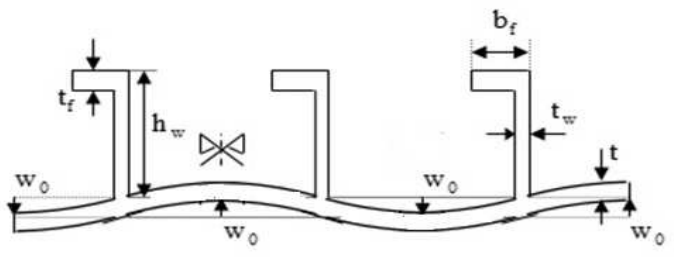

Figure 2. Geometric parameters of the panel and characteristics of the initial distortion in the xy plane

\subsection{Material properties}

The material properties considered are those of a carbon epoxy prepreg with the following properties: density $\rho=1450 \mathrm{~kg} / \mathrm{m}^{3}$, Poisson's coefficient $v=0.15$ and rigidity modules of the orthotropic elastic matrix $\mathrm{E}_{1}=139 \mathrm{GPa}, \quad \mathrm{E}_{2}=35 \mathrm{GPa}, \mathrm{G}_{12}=\mathrm{G}_{13}=50 \mathrm{GPa}$ and $\mathrm{G}_{23}=42 \mathrm{GPa}$.

The composite is made of various plies that have different orientations $\theta$. The thickness of each layer is given as $0.0015 \mathrm{~m}$. In the skin plate four symmetric layers were used and three symmetric layers for the stiffeners.

The orientations of plies in the composite are given in table 1 .

Figure 3 shows an example of the orientation set of the fibres in the composite as obtained from Abaqus CAE. The set of plies is denoted $[0,90,45,-45] \mathrm{s}$, where $\mathrm{s}$ stands for symmetric
Table 1. The orientations sets of the plies in the composite used in the simulations

\begin{tabular}{|c|c|c|}
\hline $\begin{array}{c}\text { Set of plies } \\
\text { Orientations }\end{array}$ & Skin & Stiffeners \\
\hline 1 & {$[45,0,-45,90] \mathrm{s}$} & {$[45,0,45] \mathrm{s}$} \\
\hline 2 & {$[0,90,45,-45] \mathrm{s}$} & {$[45,0,45] \mathrm{s}$} \\
\hline 3 & {$[45,0,-45,90] \mathrm{s}$} & {$[45,0,45] \mathrm{s}$} \\
\hline 4 & {$[30,-30,-30,30] \mathrm{s}$} & {$[45,0,45] \mathrm{s}$} \\
\hline 5 & {$[45,0,-45,90] \mathrm{s}$} & {$[30,-30,30] \mathrm{s}$} \\
\hline 6 & {$[0,90,45,-45] \mathrm{s}$} & {$[30,-30,30] \mathrm{s}$} \\
\hline 7 & {$[45,0,-45,90] \mathrm{s}$} & {$[-30,-30,30] \mathrm{s}$} \\
\hline 8 & {$[30,-30,-30,30] \mathrm{s}$} & {$[30,-30,30] \mathrm{s}$} \\
\hline
\end{tabular}

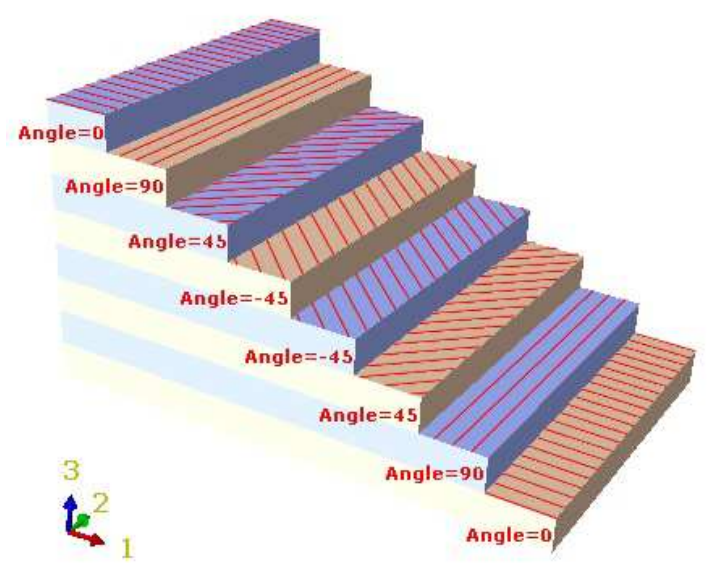

Figure 3. The orientation of the fibres in the skin of the composite in the case of $[0,90,45,-45] \mathrm{s}$.

\subsection{Buckling strength analysis}

For a given combination of parameters including sets of plies orientations and amplitude of initial imperfection, a finite element model is built under Abaqus software packages. The modelling integrates geometric nonlinearities and iterations are controlled using the Ricks method. This type of analysis is used usually to study the instability and the post-buckling behaviour of the structure and solves both the load and the displacement during the analysis using another variable which is the arc length. The buckling load is determined to be that one associated with the limit point on the curve which gives the resulting axial load as a function of the axial shortening of the panel. 


\section{Results and discussion}

To assess the influence of initial geometric imperfections on the buckling strength of composites panels, three levels for the modal imperfection amplitude were considered: $\mathrm{w}_{0}=2,4,6 \mathrm{~mm}$. Different plies orientation sets were also considered as given in Table 1 .

The buckling strength load for given plies orientations and initial geometric imperfections are summarized in Table 2.

Table 2. Buckling strength load of the composite panel as function of the plies orientations and initial imperfection amplitude

\begin{tabular}{|c|c|c|c|}
\hline $\begin{array}{l}\text { Plies } \\
\text { Set }\end{array}$ & $\mathrm{w}_{0}=2 \mathrm{~mm}$ & $\mathrm{w}_{0}=4 \mathrm{~mm}$ & $\mathrm{w}_{0}=6 \mathrm{~mm}$ \\
\hline 1 & 7115025 & 6954494 & 6571126 \\
\hline 2 & 7168455 & 6830670 & 6448324 \\
\hline 3 & 7473821 & 6909395 & 6520073 \\
\hline 4 & 7012424 & 6695725 & 6315012 \\
\hline 5 & 7473821 & 7099268 & 6607864 \\
\hline 6 & 7402314 & 6997066 & 6522682 \\
\hline 7 & 7476491 & 7109686 & 6640345 \\
\hline 8 & 7261103 & 6816493 & 6322702 \\
\hline
\end{tabular}

The results show that the orientations of the layers and the initial geometric imperfection have important effect on the buckling strength of the composite panel. Increasing the amplitude of the initial imperfection yields decrease of the buckling strength load. The most adverse combination of the orientations of layers for the composite stiffened panel which is associated to set number 4: $[30,-30,-30,30] \mathrm{s}$ for the skin plate and $[45,0,45] \mathrm{s}$ for the stiffeners. This was the case for the three values of initial imperfection amplitudes. The most favourable combination of the layer's orientation is given by the set number $7:[45,0,-45,90]$ s for the skin plate and $[-30,-30,30] \mathrm{s}$ for the stiffeners. Moreover, the buckling shapes were found to be slightly affected by the orientations and the numbers of layers of the composite panel.

\section{Conclusion}

To avoid the early collapse of the composite stiffened panel, it is preferable to take various cautions when designing the structure, in choosing the type of material and the geometry. In this work, the buckling strength of a composite stiffened panel loaded in compression was studied by using the finite element method. The effects of the different layers orientations of the fibres in the composite and the initial geometric imperfection resulting of the assembling process were taken into account. It was found that the buckling strength depends slightly on the orientations of the fibres and largely on the amplitude of initial geometric imperfections. Optimisation of the plies orientations is then possible.

\section{References}

1. A.W. Leissa, ASME Appl. Mech. Rev. 40, 575 (1987)

2. Y.X. Zhang, C.H. Yang, Composite Structures 88, 147 (2009)

3. F. Cappello, D. Tumino, Composites Science and Technology 66, 264 (2006)

4. A. Karrech, M. Elchalakani, M. Attar, A.C. Seibi, Composite Structures 174, 134 (2017)

5. K. Liang, Q. Sun, Composite Structures 168, 266 (2017)

6. O. Namdar, H. Darendeliler, Composites Part B 120, 143 (2017).

7. M. Heidari-rarani, S.S. Khalkhali-sharifi, M.M. Shokrieh, Comput. Mater. Sci. 89, 89 (2014)

8. C. Dawn, Effects of Thickness and ply orientation on buckling of laminated plates (NASA Tech. Memo. 87691, 1986)

9. P. Lengvarský, J. Bocko, M. Hagara, Am. J. Mech. Eng. 4, 413 (2016)

10. J.D. Whitcomb, K.N. Shivakumar, Journal of Composite Materials 23, 714 (1989) 